\title{
ERRATUM
}

\section{Inhibition of histone H3K9 methyltransferases by gliotoxin and related epipolythiodioxopiperazines}

Masanari Takahashi, Yasushi Takemoto, Tadahiro Shimazu, Hisashi Kawasaki, Makoto Tachibana, Yoichi Shinkai, Motoki Takagi, Kazuo Shin-ya, Yasuhiro Igarashi, Akihiro Ito and Minoru Yoshida

The Journal of Antibiotics (2014) 67, 737-738; doi:10.1038/ja.2014.101

Correction to: The Journal of Antibiotics (2012) 65, 263-265; doi:10.1038/ja.2012.6; published online 15 February 2012

Due to a typesetting error, in Figure 1, (e) and (f) were published incorrectly. Production would like to apologize for this mistake.
Also, the author would like to take the opportunity to correct the image in the Figure 1 (f).

The correct Figure 1 is shown in next page. 
a<smiles>CN1SS[C@H]2C3(CC4=CC=C[C@H](O)[C@]42C3(CO)CO)C1=O</smiles>

Gliotoxin b

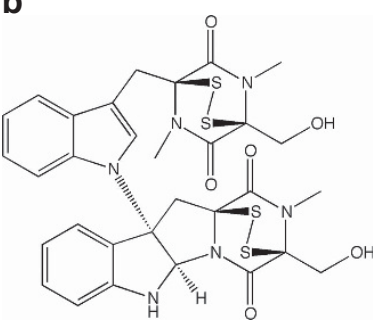

Chetomin
C

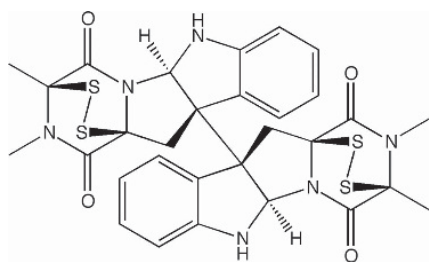

$11,11^{\prime}$-dideoxyverticillin A d<smiles>CO[C@H]1C=CC=C2C[C@@]3(SC)C(=O)N(C)C(CO)(CO)C(=O)N3[C@]21C</smiles>

Bisdethiobis(methylthio) -acetylgliotoxin e

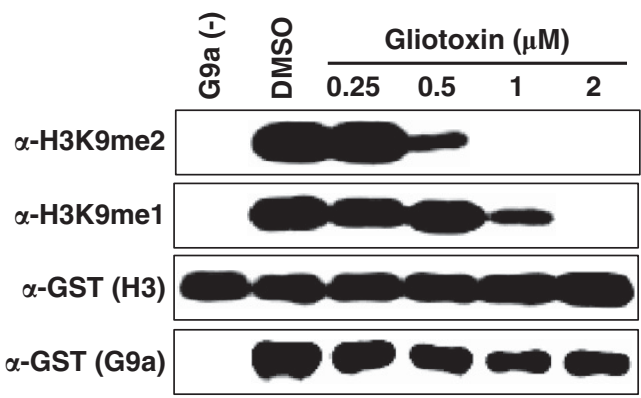

f

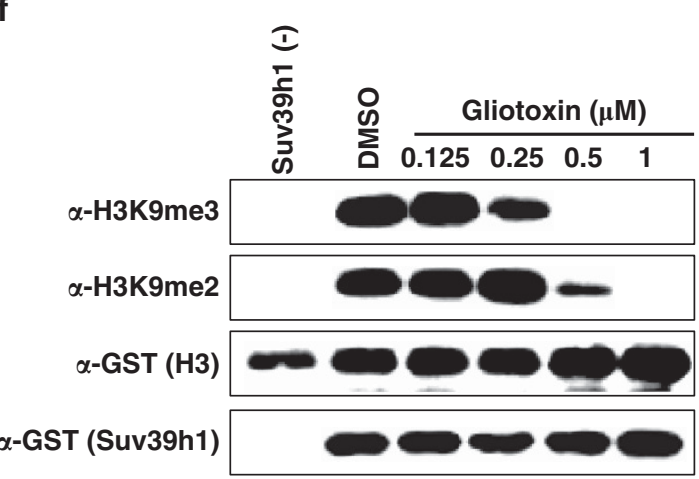

g

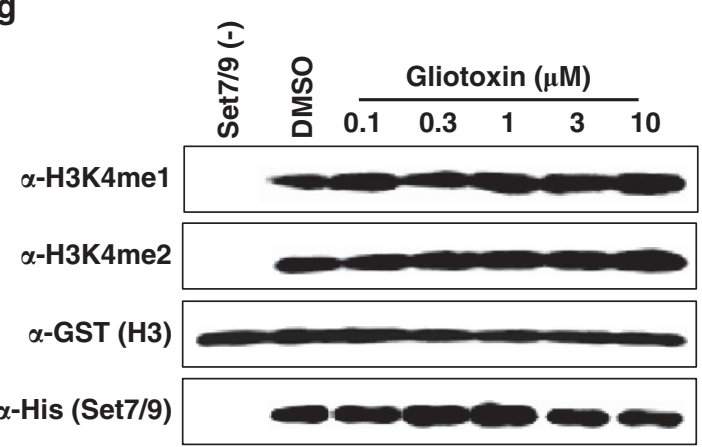

Figure 1 Gliotoxin inhibits methyltransferase activities of G9a and Suv39h1 in vitro. Structures of gliotoxin (a), chetomin (b), 11,11'-dideoxyverticillin A (c) and bisdethiobis(methylthio)-acetylgliotoxin (d). (e-g) Dose response of histone H3 methylation inhibition as a function of gliotoxin concentration. Indicated concentrations of gliotoxin were added to the methylation reaction mixture containing GST-fused histone H3 (1-57 a.a.), $10 \mu \mathrm{g} \mathrm{ml-1}$ of SAM and either GST-fused G9a (e), GST-fused Suv39h1-H320R (f) or His-tagged Set7/9 (g). GST-fused methylated histone H3 was detected by immunoblotting using an anti-H3K9me1, anti-H3K9me2 or anti-H3K9me3 antibody as indicated. Total levels of GST-fused histone H3 and methyltransferases were detected by immunoblotting using anti-GST and anti-His antibodies. 